\title{
Programa de tratamiento integrativo para la fibromialgia: estudio preliminar
}

\author{
AMANDA DÍAZ-GARCÍA \\ amdiaz@uji.es \\ GuAdALUPE MOLINARI \\ molinari@uji.es \\ ADRIANA MIRA \\ miraa@uji.es \\ MACARENA ESPINOZA \\ macaespinoza@gmail.com \\ AZUCENA GaRcía-PALACIOS \\ azucena@uji.es
}

\section{Resumen}

Uno de los síndromes de dolor crónico más prevalente en la consulta hospitalaria y ambulatoria de reumatología es la fibromialgia (FM). Los programas de tratamiento psicológicos actuales que se han desarrollado para los pacientes que sufren FM están basados en la Terapia Cognitivo Conductual (TCC). Muchos de estos tratamientos han mostrado eficacia de forma aislada en estudios empíricos en entornos de laboratorio. Por esto, es necesario probar la eficacia de estos programas de tratamiento en entornos naturales como es el caso de una Unidad de Salud Mental (USM). Para el presente estudio se ha desarrollado un programa de intervención, aplicado en una USM, dirigido no sólo a la reducción del dolor sino también a dotar al paciente de herramientas para lograr una mejor aceptación del mismo, aumentar sus emociones positivas y su calidad de vida, afrontando así de mejor manera el problema del dolor.

Se realizó un estudio piloto con el fin de valorar la eficacia preliminar de un programa de tratamiento psicológico que integra técnicas de la TCC junto con herramientas de psicología positiva, aceptación, mindfulness e hipnosis (que han mostrado evidencia en el tratamiento del dolor crónico). En el estudio participaron 5 pacientes (4 mujeres y 1 hombre) que recibieron dicho programa en 8 sesiones. Los resultados en el post-tratamiento indicaron que los pacientes aumentaron de manera significativa sus niveles de aceptación del dolor, auto-compasión y calidad de vida. Se observa también una mejora en el estado de ánimo acompañada de una reducción en la interferencia del dolor.

Nuestro trabajo aporta datos preliminares que apoyan la utilidad de incluir nuevos componentes a la hora de abordar la FM.

Palabras clave: dolor crónico, fibromialgia (FM), Terapia Cognitivo Conductual (TCC), Aceptación, Hipnosis. 


\section{Abstract}

One of the most prevalent chronic pain syndromes in the hospital and outpatient rheumatology clinic is fibromyalgia (FM). The current psychological treatment programs that have been developed for patients suffering from FM are based on Cognitive Behavioral Therapy (CBT). Many of these treatments have shown efficacy in isolation in empirical studies in laboratory settings. Therefore, it is necessary to test the effectiveness of these treatment programs in natural settings such as a Mental Health Unit (USM). For the present study, an intervention program has been developed, applied at a USM, aimed not only at reducing pain but also at providing the patient with tools to achieve better acceptance of it, increase their positive emotions and their quality of life, therefore addressing better the problem of pain.

A pilot study was conducted to assess the preliminary efficacy of a psychological treatment program that integrates CBT techniques along with positive psychology, acceptance, mindfulness and hypnosis tools (which have shown evidence in the treatment of chronic pain). The study involved 5 patients (4 women and 1 man) who received an 8 session program. Post- treatment results indicate that patients significantly increased their levels of pain acceptance, self-pity, and quality of life. There is also an improvement in mood accompanied by a reduction in pain interference.

Our work provides preliminary data that support the usefulness of including new components when addressing FM.

Key Words: Chronic pain, fibromyalgia (FM), Cognitive Behavioral Therapy (CBT), Acceptance, Hypnosis.

\section{Introducción}

La Fibromialgia (FM) se define como un síndrome de dolor crónico de etiología desconocida (Wolfe y cols., 2011) que se caracteriza por dolor musculoesquelético generalizado acompañado de otros síntomas como la fatiga intensa o las alteraciones del sueño (Bennett, Jones, Turk, Russell, y Matallana, 2007). Así mismo, los pacientes con FM, pueden presentar otras manifestaciones clínicas comunes como la rigidez articular, cefaleas tensionales, parestesias en extremidades o sensación de hinchazón o tumefacción en las manos (Alegre de Miquel, 2006). Esta heterogeneidad de síntomas suele coexistir también con trastornos cognitivos o con la comorbilidad de trastornos del estado de ánimo y trastornos de ansiedad (Fietta, 2004).

La FM constituye un importante problema de salud pública, con una prevalencia que oscila entre el 2 y el $4 \%$ en la población general (Mease, 2005) y es considerada como uno de los síndromes de dolor crónico más prevalentes en la consulta hospitalaria y ambulatoria de reumatología (Nampiaparampil y Shmerling, 2004). En España, la FM afecta al $2.4 \%$ de la población general mayor de 20 años, siendo más frecuente en mujeres (4.2\%) que en hombres $(0.2 \%)$, lo que supone una relación mujer:varón de 21:1 (Mas, Carmona, Valverde, y Ribas, 2008). Además, la FM está asociada con frecuentes repercusiones laborales, resultando en una alta carga tanto económica como social (Spaeth, 2009).

En la actualidad no se conocen con precisión las causas de la FM aunque existen numerosos estudios que han investigado sobre los posibles mecanismos patogénicos que actúan en esta enfermedad. Algunos de estos estudios muestran la existencia de una alteración en 
los mecanismos de procesamiento del dolor debido a posibles desequilibrios en los neuromoduladores del sistema nervioso central (Crofford, 2005), lo que conlleva a que estos pacientes tengan un umbral más bajo hacia el dolor (Price y Staud, 2005).

Los criterios diagnósticos de la FM se establecieron inicialmente en 1990 (Wolfe y cols., 1990) y señalaban una historia de dolor generalizado de más de 3 meses de duración así como múltiples puntos sensibles al dolor (al menos 11 de 18 puntos simétricos: occipital, cervical bajo, trapecio, supraespinoso, segundo espacio intercostal en la unión costocondral, epicóndilo, glúteo, trocánter mayor y rodilla). Actualmente, estos criterios se han modificado y se han propuesto para su diagnóstico el Índice de dolor generalizado (Widespread Pain Index; WPI) y una Escala de gravedad de síntomas (Symptom Severity Score; SS-Score) (Wolfe y cols., 2011), los cuales se basan en la valoración de los principales síntomas y en la información aportada por parte de los pacientes.

Debido a toda la complejidad de síntomas y manifestaciones, el tratamiento interdisciplinario y multicomponente se ha considerado como el tratamiento de elección para la FM, con el objetivo de mejorar la sintomatología del paciente, proporcionar un cuidado de carácter integral y mejorar, en definitiva, la calidad de vida de estas personas (Otis, 2013). Por esta razón, los mayores beneficios en el tratamiento de las personas que sufren FM se logra cuando se combinan diferentes enfoques y tratamientos con un abordaje amplio de profesionales como médicos, especialistas, terapeutas físicos y psicólogos (Gatchel, Peng, Peters, Fuchs, y Turk, 2007). El tratamiento interdisciplinar incluye el tratamiento farmacológico para disminuir el dolor y mejorar el sueño, programas de ejercicio físico moderado para estirar los músculos y mejorar la capacidad cardiovascular y tratamiento psicológico, el cual se aplica a través de una fase educacional (informando al paciente sobre la naturaleza del trastorno), una fase de adquisición de habilidades (mediante el aumento de actividades significativas, técnicas de relajación para aliviar la tensión muscular y la ansiedad, técnicas de solución de problemas, estrategias de afrontamiento comportamental para hacer frente al dolor, etc.) y una fase de puesta en práctica en la que el paciente aplica todo lo aprendido para manejar los síntomas del dolor (Alegre de Miquel, 2006).

Como se ha mencionado anteriormente, dentro de esta perspectiva multidisciplinar, los programas psicológicos juegan un papel fundamental y prometedor para el tratamiento de la FM. En concreto, la Terapia Cognitivo Conductual (TCC) ha demostrado ser eficaz en la reducción del dolor y la fatiga, así como en el alivio del malestar emocional o la mejora del bienestar (Friedberg y Jason, 2001). La TCC tiene como objetivo la modificación de los esquemas de pensamiento y emociones desadaptativos así como de las emociones negativas y repertorios de afrontamiento que el individuo posee para manejar el dolor (Sullivan, Feuerstein, Gatchel, Linton, y Pransky, 2005). En un importante meta-análisis realizado para comprobar la eficacia de los tratamientos psicológicos en la FM (Glombiewski y cols., 2010) se obtuvo que la TCC se asoció con los mayores tamaños del efecto. Los autores concluyeron que la TCC fue significativamente mejor que otros tratamientos psicológicos en la reducción del dolor en la FM tanto a corto como a largo plazo, aunque continúan señalando la necesidad de realizar más estudios de eficacia ya que existen relativamente pocos estudios en el campo de los tratamientos psicológicos para la FM.

Pese a la evidencia mostrada de la TCC para la FM, muchos de estos tratamientos han probado eficacia de forma aislada en estudios empíricos, generalmente en entornos de laboratorio y no suelen ser instaurados en los servicios de atención médica. Por esta razón, es necesario poner a prueba la eficacia de estos programas de tratamiento en entornos más naturales como es el caso de una Unidad de Salud Mental (USM), en el que los pacientes acuden derivados por los servicios de atención primaria. 
Por otro lado, en los últimos años se ha enfatizado la incorporación de otras herramientas psicológicas novedosas que, junto con las técnicas de la TCC, han demostrado ser eficaces en el tratamiento de personas que sufren FM, como es el caso de la hipnosis, la aceptación o el mindfulness (Moix y Casado, 2011).

Para el presente estudio se ha desarrollado un programa de intervención psicológico, aplicado en una USM, dirigido no sólo a la reducción del dolor sino también a dotar al paciente de herramientas para lograr una mejor aceptación del mismo, aumentar sus emociones positivas y su calidad de vida, afrontando así de mejor manera el problema del dolor.

El principal objetivo de este estudio piloto es describir el programa de tratamiento y mostrar datos preliminares de los pacientes que participaron en dicha intervención. La hipótesis marcada es que a través de esta intervención se producirá una reducción significativa en las variables clínicas medidas, mostrando con ello la importancia de implantar un programa basado en TCC, pero incluyendo otras herramientas psicológicas eficaces para la FM, en una USM y desde un abordaje multidisciplinar.

\section{Método}

\section{Participantes}

La muestra estuvo compuesta por 5 pacientes (4 mujeres y 1 hombre) con diagnóstico de FM, de entre 39 y 67 años de edad. La media de edad era de 50,80 años (DT=10,50). La mayoría de los participantes estaban casados $(n=4 ; 80 \%)$ y sólo un participante era viudo ( $n$ $=1 ; 20 \%$ ). En cuanto al nivel de estudios, un participante tenía menos de 8 años de estudios $(20 \%)$, otro participante tenía un nivel de estudios primarios $(20 \%)$ y el resto presentaba nivel de estudios superiores $(n=3 ; 60 \%)$.

Entre los criterios de inclusión para recibir dicho tratamiento se encontraban: a) tener entre 18-70 años; b) tener diagnóstico de FM por un reumatólogo de acuerdo con los criterios del Colegio Americano de Reumatología (Wolfe y cols., 2011); c) no padecer esquizofrenia, trastorno bipolar, discapacidad intelectual y/o abuso/dependencia de sustancias.

\section{Instrumentos de evaluación}

Escala de Medida de la Autocompasión (Self-Compasion Scale, SCS; Neff, 2016). Se trata de una escala que cuenta con dos versiones, una larga de 26 ítems y una corta de 12 ítems. La escala fue desarrollada para representar explícitamente los pensamientos, emociones y comportamientos asociados con los diversos componentes de la autocompasión. Las respuestas se dan en una escala de 5 puntos donde 1 es «casi nunca» y 5 «casi siempre». Existe una amplia evidencia de la fiabilidad y validez de esta escala así como alta consistencia interna (Allen, Goldwasser, y Leary, 2012).

Cuestionario de Aceptación del Dolor (Chronic Pain Acceptance Questionnaire, CPA; McCracken, Vowles, y Eccleston, 2004; versión española: Menéndez, García, y Viejo, 2010). Consta de 20 ítems que evalúan la aceptación del dolor en personas que sufren dolor crónico. Con este cuestionario se obtienen tres puntuaciones: una total, y otras dos subescalas (implicación en las actividades (IA) y apertura al dolor (AD). Estas subescalas han mostrado datos de consistencia interna hallados mediante el alpha de Cronbach (Cronbach, 1951) de .82 para la primera y .78 para la segunda. 
Índice de Calidad de Vida (Quality of Lifelndex, QLI; Mezzich y cols., 2000). Se trata de un autoinforme que mide el nivel de calidad de vida percibido por el paciente en la actualidad. Consta de 10 de ítems con formato de respuesta de opción múltiple tipo Likert en una escala que puntúa de 1 («malo») a 10 («excelente»). Cuenta con buenas propiedades psicométricas (Mezzich y cols., 2000).

Inventario de Depresión de Beck (Inventory on Beck's depression, BDI-II; versión española: Sanz, Navarro, y Vázquez, 2003). Instrumento de autoinforme diseñado para evaluar la gravedad de la sintomatología depresiva. Consta de 21 ítems cada una con cuatro alternativas ordenadas de menor a mayor gravedad (de 0 a 3). Este cuestionario ha mostrado tener buenas propiedades psicométricas con una elevada consistencia interna (coeficiente de alfa de .89) (Sanz y cols., 2003).

Cuestionario de Impacto de la Fibromialgia (Fibromyalgia Impact Questionnaire, FIQ; Burckhardt, Clark, y Bennett, 1991; versión española: (Esteve-Vives, Rivera, Salvat, de Gracia, y Alegre, 2007). Es una de las medidas de autoinforme más utilizadas en el estudio de la FM. Consta de 10 preguntas medidas en una escala tipo Likert de 0 («siempre») a 3 («nunca») y que evalúan: la capacidad de realizar ciertas tareas, el número de días que se sintió bien y el número de días que no pudo realizar el trabajo, la interferencia del dolor, la fatiga, el cansancio por la mañana, la rigidez, la ansiedad y la depresión. Es un instrumento ampliamente validado (Bennett, 2005).

\section{Procedimiento}

Los participantes del estudio eran usuarios de la USM del Centro de Salud de Rafalafena de Castellón. Todos ellos fueron derivados por sus propios psiquiatras tras haberse considerado su inclusión en el estudio de tratamiento psicológico y su posible beneficio del mismo. Tras la explicación del curso del tratamiento, cada uno de ellos firmó un consentimiento informado donde mostraban acuerdo y conformidad con el programa de tratamiento en el que iban a participar. Los participantes formaron finalmente un grupo de tratamiento de 5 pacientes y contaban con dos terapeutas (terapeuta principal y co-terapeuta) que preparaban y aplicaban el tratamiento cada semana. El protocolo de evaluación se aplicó durante el pre-tratamiento y el post-tratamiento, donde los participantes cumplimentaban todos los autoinformes relacionados con el dolor y con el estado de ánimo.

El tratamiento psicológico grupal se desarrolló en colaboración con el Grupo de Investigación LabPsiTec del Departamento de Psicología Básica, Clínica y Psicobiología de la Universitat Jaume I de Castellón.

\section{Tratamiento}

Se trata de un programa de tratamiento psicológico que integra técnicas de la TCC junto con herramientas de psicología positiva, aceptación, mindfulness e hipnosis, las cuales han mostrado evidencia en el tratamiento del dolor crónico (Moix y cols., 2011). El programa se desarrolló en 8 sesiones semanales de una duración aproximada de 2 horas y aplicadas en formato grupal. Cada una de las sesiones se llevó a cabo en la sala de preparación al parto de la USM de Rafalafena, la cual reunía todas las necesidades requeridas en cada sesión. Una descripción más detallada de este tratamiento multicomponente puede observarse en la tabla 1. 
Tabla 1

Componentes terapéuticos del programa

\begin{tabular}{ll}
\hline Componentes & Objetivos \\
\hline Psicoeducación & Explicación de la enfermedad teniendo en cuenta \\
& aspectos médicos, psicológicos y sociales relacionados. \\
& Explicación de las normas de la terapia grupal y de la \\
& base lógica del tratamiento. \\
\hline
\end{tabular}

Aceptación radical

Reconocimiento de las propias limitaciones físicas y cambios de hábitos. Aprender a estar en contacto con la propia experiencia, incluso cuando ésta no es agradable, y aceptarla tal y como es. Aceptación de mi nuevo «yo».

Auto-hipnosis

Reducción de la tensión muscular, los problemas de sueño, el estrés emocional, etc., a través de diferentes técnicas de relajación.

Programación de actividades

Aumentar el número e intensidad de emociones positivas a través de un nivel de actividad adecuado para afrontar el dolor. Realizar actividades significativas para el individuo.

Flexibilidad cognitiva

Abordar y modificar los pensamientos desadaptativos, aprendiendo a generar otras posibles interpretaciones alternativas a las distintas situaciones.

Habilidades de comunicación

Aprender a identificar los principales problemas de comunicación para dirigirse hacia una comunicación más eficaz. Mejorar las relaciones interpersonales, la autoestima y poner en práctica la asertividad.

Auto-compasión

Aprender la necesidad de cuidarnos, de ser amables con nosotros mismos, buscando el bienestar y el alivio del sufrimiento.

Prevención de recaídas

Repasar todas las habilidades aprendidas con el propósito de revisar los contenidos y ver la manera de mantener y continuar con los progresos logrados hasta el momento. Valorar la forma de actuar en situaciones futuras de riesgo.

\section{Resultados}

En la Tabla 2 se muestran las medias, desviaciones típicas y la significación estadística de todas las variables clínicas. Los cambios tras la intervención se midieron a través de la prueba no paramétrica de los signos de Wilcoxon para la comparación entre las variables medidas en el pre-tratamiento y en el post-tratamiento. 
Tabla 2

Efectos de la intervención en las distintas variables clínicas

\begin{tabular}{lllllll}
\hline \multirow{2}{*}{ VARIABLE } & \multicolumn{5}{c}{ Pre } & \multicolumn{5}{c}{ Post } & \multirow{2}{*}{ Z } \\
\cline { 2 - 5 } & Media & DT & Media & DT & \\
\hline SCS & 2,25 & 0,63 & 3,20 & 0,45 & $-2,03$ & $0,04^{*}$ \\
\hline CPAQ & 39,40 & 21,65 & 55,20 & 10,57 & $-2,02$ & $0,04^{*}$ \\
\hline QLI & 4,48 & 1,79 & 6,04 & 1,47 & $-2,04$ & $0,04^{*}$ \\
\hline BDI-II & 30,83 & 9,79 & 20,40 & 9,96 & $-1,76$ & 0,08 \\
\hline FIQ & 78,70 & 7,33 & 69,95 & 23,70 & $-0,99$ & 0,35 \\
\hline
\end{tabular}

Nota. $\mathrm{M}=$ media; $\mathrm{DT}=$ desviación típica; Pre = Pre-tratamiento; Post= Post- tratamiento; $\mathrm{SCS}=$ Self Compasssion Scale; $\mathrm{CPAQ=}$ Chronic Pain Acceptance Questionnaire; QLI= Quality of Life Index; BDI-II= Inventory on Beck's depression; FIQ= Fibromyalgia Impact Questionnaire.

Como muestran los resultados, pueden observarse diferencias estadísticamente significativas del pre-tratamiento al post-tratamiento en las variables señaladas $\left(^{*}\right)(p<0.05)$. Esto demuestra que los pacientes aumentaron de manera significativa sus niveles de aceptación del dolor, de auto-compasión y sus niveles de calidad de vida tras la intervención. Así mismo, los resultados obtenidos se encaminan en la dirección de lo esperado observándose también mejoras en el estado de ánimo acompañada de una reducción en la interferencia del dolor, aunque éstas no llegan a ser estadísticamente significativas.

\section{Discusión y conclusiones}

El objetivo de este estudio piloto fue describir un programa de tratamiento novedoso para la FM que integra distintas técnicas psicológicas que han mostrado ser eficaces y presentar datos preliminares de un grupo de pacientes que participaron en dicha intervención.

Teniendo en cuenta la hipótesis planteada al comienzo del estudio, podemos decir que contamos con evidencia preliminar a su favor. Los resultados mostraron que a través de esta intervención se produjo un aumento significativo en los niveles de aceptación del dolor, calidad de vida y auto-compasión. Esto es importante ya que se observan cambios que podrían estar vinculados justamente a aquellos módulos de tratamiento más novedosos, como son el de aceptación radical y de autocompasión, que parecen haber producido cambios significativos en dichas variables. La aceptación del dolor no sólo ha sido estudiada en estudios previos como una importante variable de resultado, sino que también se ha encontrado que es un importante mecanismo de cambio para alcanzar la reducción sintomática en pacientes con FM (Luciano y cols., 2014). En cuanto a la auto-compasión, estudios previos han demostrado la relación entre esta variable y las variaciones en el dolor musculoesquelético (Wren y cols., 2012), sin embargo esta relación no había sido estudiada previamente en pacientes con FM. 
Si bien en el presente estudio no es posible aislar el efecto diferencial de cada uno de los componentes de tratamiento, los cambios en estas variables resultan prometedores. Además, si bien los cambios no fueron estadísticamente significativos, encontramos reducciones en la interferencia producida por el dolor y un aumento en el estado de ánimo.

En cuanto a las limitaciones del presente estudio, es preciso destacar que se trata de un estudio piloto, con un número reducido de participantes, de manera que los resultados deben tomarse con precaución. Además, como se mencionaba anteriormente, con el presente diseño no fue posible aislar los efectos de cada uno de los componentes. Es necesario que futuros estudios pongan a prueba este protocolo de tratamiento en un estudio controlado e incorporando medidas de evaluación que sean capaces de identificar módulo a módulo los cambios alcanzados por los pacientes. En este sentido, en el futuro sería interesante a llevar a cabo estudios con un diseño de desmantelamiento (Papa, Follette, Papa, y Follette, 2015). Este diseño tiene como objetivo investigar terapias que tienen múltiples componentes con el fin de identificar las características de la terapia que son los mecanismos activos de cambio o identificar el grado en que los componentes específicos aumentan la magnitud del cambio atribuible a otros componentes (Papa y cols., 2015).

Por todo lo dicho anteriormente, es posible decir que este programa de tratamiento integrativo (basado en TCC pero que incluye otras herramientas psicológicas eficaces para la FM) no sólo es viable de implementar en una USM desde un abordaje multidisciplinar, sino que además ha demostrado su eficacia preliminar a la hora de promover mejoras significativas en la calidad de vida de personas con FM.

\section{Referencias bibliográficas}

Alegre de Miquel, C. (2006). I Simposio de Dolor en Reumatología. Reumatología Clínica, 2(1), 55-66.

Allen, A. B., Goldwasser, E. R. y Leary, M. R. (2012). Self-compassion and Well-being among Older Adults. Self and Identity, 11(4), 428-453.

Bennett, R. (2005). The Fibromyalgia Impact Questionnaire (FIQ): A review of its development, current version, operating characteristics and uses. Clinical and Experimental Rheumatology, 23(5 SUPPL. 39), S154-62.

Bennett, R. M., Jones, J., Turk, D. C., Russell, I. J. y Matallana, L. (2007). An internet survey of 2,596 people with fibromyalgia. BMC Musculoskeletal Disorders, 8, 27.

Burckhardt, C. S., Clark, S. R. y Bennett, R. M. (1991). The fibromyalgia impact questionnaire: development and validation. The Journal of Rheumatology, 18(5), 728-33.

Crofford, L. J. (2005). The relationship of fibromyalgia to neuropathic pain syndromes. The Journal of Rheumatology Supplement, 75.

Cronbach, L. J. (1951). Coefficient alpha and the internal structure of tests. Psychometrika, 16(3), 297-334.

Esteve-Vives, J., Rivera Redondo, J., Isabel Salvat Salvat, M., de Gracia Blanco, M. y de Miquel, C. A. (2007). Propuesta de una versión de consenso del Fibromyalgia Impact Questionnaire (FIQ) para la población española. Reumatología Clínica, 3(1), 21-24.

Fietta, P. (2004). Fibromyalgia: state of the art. Minerva Medica, 95(1), 35-47, 47-52.

Friedberg, F. y Jason, L. A. (2001). Chronic fatigue syndrome and fibromyalgia: clinical assessment and treatment. Journal of Clinical Psychology, 57(4), 433-55.

Gatchel, R. J., Peng, Y. B., Peters, M. L., Fuchs, P. N. y Turk, D. C. (2007). The biopsychosocial approach to chronic pain: Scientific advances and future directions. Psychological Bulletin, 133(4), 581-624. 
Glombiewski, J. A., Sawyer, A. T., Gutermann, J., Koenig, K., Rief, W. y Hofmann, S. G. (2010). Psychological treatments for fibromyalgia: A meta-analysis. Pain, 151(2), 280-295.

Luciano, J. V., Guallar, J. A., Aguado, J., López-del-Hoyo, Y., Olivan, B., Magallón, R., ... Garcia-Campayo, J. (2014). Effectiveness of group acceptance and commitment therapy for fibromyalgia: A 6-month randomized controlled trial (EFFIGACT study). PAIN®, 155(4), 693-702.

Mas, A. J., Carmona, L., Valverde, M. y Ribas, B. (2008). Prevalence and impact of fibromyalgia on function and quality of life in individuals from the general population: results from a nationwide study in Spain. Clinical and Experimental Rheumatology, 26(4), 519-26.

McCracken, L. M., Vowles, K. E. y Eccleston, C. (2004). Acceptance of chronic pain: component analysis and a revised assessment method. Pain, 107(1-2), 159-66.

Mease, P. (2005). Fibromyalgia syndrome: review of clinical presentation, pathogenesis, outcome measures, and treatment. The Journal of Rheumatology. Supplement, 75, 6-21.

Menéndez, A. G., García, P. F. y Viejo, I. T. (2010). Aceptación del dolor crónico en pacientes con fibromialgia: adaptación del Chronic Pain Acceptance Questionnaire(CPAQ) a una muestra española. Psicothema, 22(4), 997-1003.

Mezzich, J. E., Ruipérez, M. A., Pérez, C., Yoon, G., Liu, J. y Mahmud, S. (2000). The Spanish version of the quality of life index: presentation and validation. The Journal of Nervous and Mental Disease, 188(5), 301-5.

Moix, J., Casado, I. y Del Manual Del Dolor, C. (2011). Terapias Psicológicas para el Tratamiento del Dolor Crónico Psychological Therapies to Treat Chronic Pain. Clínica Y Salud, 22(1), 41-50.

Nampiaparampil, D. E., y Shmerling, R. H. (2004). A review of fibromyalgia. The American Journal of Managed Care, 10(11 Pt 1), 794-800.

Neff, K. D. (2016). The Self-Compassion Scale is a Valid and Theoretically Coherent Measure of Self-Compassion. Mindfulness, 7(1), 264-274.

Otis, J. D. (2013). Flor, H., \&amp; Turk, D. C. (2011)Chronic Pain: An Integrated Biobehavioral Approach Seattle: IASP Press. Cognitive and Behavioral Practice.

Papa, A., Follette, W. C., Papa, A. y Follette, W. C. (2015). Dismantling Studies of Psychotherapy. In The Encyclopedia of Clinical Psychology (pp. 1-6). Hoboken, NJ, USA: John Wiley \& Sons, Inc.

Price, D. D. y Staud, R. (2005). Neurobiology of fibromyalgia syndrome. The Journal of Rheumatology Supplement, 75.

Sanz Fernández, J., Navarro, M. E. y Vázquez Valverde, C. (2003). Adaptación española del Inventario para la Depresión de Beck-II (BDI-II): propiedades psicométricas en estudiantes universitarios. Análisis Y Modificación de Conducta, ISSN-E 0211-7339, Vol. 29, Nº. 124, 2003, Págs. 239-288, 29(124), 239-288.

Spaeth, M. (2009). Epidemiology, costs, and the economic burden of fibromyalgia. Arthritis Research \& Therapy, 11(3), 117.

Sullivan, M. J., Feuerstein, M., Gatchel, R., Linton, S. J. y Pransky, G. (2005). Integrating Psychosocial and Behavioral Interventions to Achieve Optimal Rehabilitation Outcomes. Journal of Occupational Rehabilitation, 15(4), 475-489.

Wolfe, F., Clauw, D. J., Fitzcharles, M. A., Goldenberg, D. L., Häuser, W., Katz, R. S., ...Winfield, J. B. (2011). Fibromyalgia criteria and severity scales for clinical and epidemiological studies: A modification of the ACR preliminary diagnostic criteria for fibromyalgia. Journal of Rheumatology, 38(6), 1113-1122.

Wolfe, F., Smythe, H. A., Yunus, M. B., Bennett, R. M., Bombardier, C., Goldenberg, D. L., ... Clark, P. (1990). The American College of Rheumatology 1990 Criteria for the Classifica- 
tion of Fibromyalgia. Report of the Multicenter CriteriaCommittee. Arthritis and Rheumatism, 33(2), 160-72.

Wren, A. A., Somers, T. J., Wright, M. A., Goetz, M. C., Leary, M. R., Fras, A. M., .. Keefe, F. J. (2012). Self-Compassion in Patients With Persistent Musculoskeletal Pain: Relationship of Self-Compassion to Adjustment to Persistent Pain. Journal of Pain and Symptom Management, 43(4), 759-770. 